\title{
Cuerpo extraño bronquial. Experiencia de los últimos 18 años en Hospital Guillermo Grant Benavente, Concepción, Chile
}

\author{
Bronchial foreign body. Experience of the last 18 years in \\ Hospital Guillermo Grant Benavente, Concepcion, Chile
}

Ignacio Mendoza A $^{1}$, Gino Marisio Lㄹ, Sahba Sedaghat N

\begin{abstract}
RESUMEN
Introducción: Los cuerpos extraños en vía aérea (CEVA) continúan siendo un desafío clínico que genera un espectro de presentaciones, desde mínima sintomatología a compromiso respiratorio, falla respiratoria e incluso muerte.

Objetivos: Describir y analizar las características epidemiológicas y clínicas de los pacientes con sospecha de CEVA evaluados en el Hospital Guillermo Grant Benavente.

Material y método: Estudio retrospectivo y descriptivo de pacientes con diagnóstico de CEVA admitidos en nuestro servicio de urgencia, entre los años 1997 y 2015. Se analizaron diferentes variables, especialmente en relación a síntomas, signos, hallazgos radiológicos, complicaciones, tratamiento y resultados.

Resultados: Se encontraron 117 broncoscopías rígidas, confirmándose CEVA en 94 (80,4\%). La mayoría niños entre 1-3 años. Hubo más de una consulta previa al diagnóstico en el $29,8 \%$, siendo la mayoría de las consultas precoces. La mayoría informó síndrome de penetración, pero esto no hizo la diferencia en el diagnóstico. El 80,9\% de los casos confirmados tuvo alguna alteración radiológica (excluyendo cuerpos radioopacos) $(p<0,05)$. Se ubicó en árbol bronquial derecho mayormente, siendo de una naturaleza variada. Se extrajo en el primer intento sin incidentes el 91,5\%. No hubo complicaciones reportadas en relación a las extracciones.

Conclusiones: Un cuerpo extraño en el tracto respiratorio es una emergencia quirúrgica, pudiendo ser incluso fatal. Un alto índice de sospecha es fundamental para evitar complicaciones.
\end{abstract}

Palabras clave: Cuerpo extraño bronquial, vía aérea, broncoscopía rígida.

\section{ABSTRACT}

Introduction: Airway Foreign bodies (AFB) remain a clinical challenge, generate a spectrum of presentations, from minimal symptoms to respiratory distress, respiratory failure and even death.

Aim: To describe and analyze the epidemiological and clinical characteristics of patients with suspected $A F B$.

Médico Servicio de Otorrinolaringología y Cirugía de Cabeza y Cuello, HGGB. 
Material and methods: A retrospective and descriptive study of patients diagnosed with AFB admitted to our Emergency Service between 1997 and 2015. Different variables were analyzed, especially their symptoms, signs, radiologic findings, complications, treatment and outcomes.

Results: 117 rigid bronchoscopies associated with suspected AFB were found. The diagnosis was confirmed in 94 cases (80.4\%). Most of them were children between 1-3 years. There was more than one consultation before the diagnosis in 29.85. Most of the patients reported penetration syndrome, but this made no difference on diagnosis. $80.9 \%$ of the confirmed cases had some radiological alteration (excluding radiopaque bodies) ( $p<0.05)$. Most of the cases were found in the right bronchial tree and were of a varied nature. They were extracted in the first attempt without incidents in $91.5 \%$ of the cases. There were no reported complications regarding extractions.

Conclusions: A foreign body in the respiratory tract is a surgical emergency, and may even be fatal. A high index of suspicion is essential to avoid complications.

Key words: Bronchial foreign body, bronchial airway, rigid bronchoscopy.

\section{INTRODUCCIÓN}

El CEVA es un grave problema que se presenta en lactantes y preescolares, menos frecuentemente en adultos (20\%), y que es potencialmente mortal ${ }^{1-4}$. Hasta el término de esta revisión continúa siendo la principal causa de muerte de origen otorrinolaringológico en Chile, y el $7 \%$ de las muertes accidentales en menores de cuatro años en Estados Unidos ${ }^{6}$. Se desprende de esto que la edad en los niños es el principal factor de riesgo, fundamentalmente dado por la dentición inmadura de éstos que no les permite morder adecuadamente, la actividad física durante la alimentación y sobre todo la tendencia a explorarlo todo a través de su boca.

Los cuerpos extraños aspirados más comunes son de origen vegetal, siendo el maní el más importante en nuestro medio ${ }^{2}$, seguido por una larga lista de objetos tanto orgánicos como inorgánicos. La aparición del síndrome de penetración o sofocación en un niño previamente sano, muy bien descrito por $\mathrm{Otte}^{2}$, es un síntoma bastante elocuente de aspiración de un CE. No sólo importa la naturaleza o ubicación del CE, sino que la permanencia de éste en la vía aérea jugará un papel crucial en la sobrevida del paciente. En relación al tratamiento, si la broncoscopía rígida falla en la extracción y el paciente se torna clínicamente inestable, debemos tener siempre en mente la necesidad de realizar una traqueostomía de rescate, y en casos seleccionados incluso puede ser necesaria incluso la toracotomía.

Los CEVA han sido ampliamente reportados alrededor del mundo, y así también es como varía su frecuencia, dependiendo de las condiciones locales, la variedad de la comida y los sistemas de salud disponibles, entre otros factores.

La presente revisión busca actualizar la epidemiología del CE aspirado en nuestro medio y la utilidad de la broncoscopía rígida (BR) como método de diagnóstico y terapéutico.

\section{OBJETIVOS}

Describir y analizar las características epidemiológicas y clínicas de los pacientes con sospecha de CEVA que se presentan en el Hospital Guillermo Grant Benavente de Concepción, desde su diagnóstico hasta su tratamiento con BR.

\section{MATERIAL Y MÉTODO}

Se realizó un estudio de diseño observacional, retrospectivo y descriptivo. Se revisaron las historias clínicas de todos los pacientes sometidos a revisión de vía aérea mediante $B R$ por sospecha de cuerpo extraño en vía aérea, que consultaron en el Hospital Guillermo Grant Benavente de Concepción durante el periodo de agosto 1996 a octubre 2015.

Se consignaron datos demográficos, comorbilidades, síntomas y signos, tiempo de evolución al ingreso hospitalario, número de consultas hasta el diagnóstico, hallazgos imagenológicos, características de los cuerpos extraños extraídos y su 
ubicación en la vía aérea, tratamiento efectuado y evolución del paciente, entre otras variables.

El análisis estadístico se llevó a cabo con el software SPSS 23 y se consideró como significativo los valores de $p<0,05$.

\section{RESULTADOS}

Se encontraron 117 BR asociadas a sospecha de CEVA, siendo positivas (cuerpo extraño presente) en 94 casos $(80,4 \%)$.

En cuanto al proceso diagnóstico, $29,8 \%$ consultó más de una vez en un centro de atención de urgencia previo a confirmarse el CEVA mediante broncoscopía. En relación al cuadro clínico, Io más frecuentemente reportado fue tos $(58,5 \%)$, disnea $(34 \%)$, fiebre $(18 \%)$, requerimiento de $\mathrm{O}_{2}$ suplementario (19\%). No hubo diferencias estadísticamente significativas con los casos donde se descartó el CEVA. El 74,5\% de los casos confirmados presentó un claro síndrome de sofocación, mientras que $69,6 \%$ de los descartados también lo refirió, no existiendo diferencias significativas. Diferente fue el caso para las radiografías de tórax, donde el $80,9 \%$ de los casos confirmados (radiolúcidos) versus $39,1 \%$ de los descartados presentó alguna alteración radiológica (atelectasia, hiperinsuflación pulmonar, neumonía, infiltrado pulmonar), lo que sí generó un $p<0,05$, con un valor predictivo positivo de $89 \%$ y un valor predictivo negativo del $47 \%$. La mayoría de los casos confirmados fueron objetos radiolúcidos (74\%). Finalmente, el diagnóstico se hizo antes de las 24 hrs en el $53 \%$ de los casos y la mayoría de los restantes se realizó durante los siguientes días (Figura 1).

Los casos de CEVA se concentraron principalmente entre los doce meses y los once años (10 meses - 63 años) siendo tres veces más frecuente en el sexo masculino (Tabla 1). La mayoría (59\%) fueron de naturaleza orgánica, especialmente en el grupo de 1-3 años $(p<0,05)$, siendo la moda el maní (el detalle de los CEVA encontrados en Tabla 2).

La ubicación fue variada. Predominaron en el árbol bronquial derecho con $60,7 \%$ versus $33 \%$ en el izquierdo, no siendo significativa esta diferencia. Destaca un caso donde se encontraron dos CEVA en el mismo paciente, uno en bronquio fuente derecho y el segundo en un bronquio lobar ipsilateral (Figura 2).

En relación al tratamiento, la extracción mediante BR fue exitosa en el primer intento para el $91,5 \%$ de los casos, $7,4 \%$ en un segundo intento, siendo necesaria la extracción mediante toracotomía en el restante 3,3\%. Se realizaron traqueos-

Tabla 1. Distribución por edad y sexo

\begin{tabular}{|lrrrl|}
\hline Edad $(\mathrm{a})$ & $\mathrm{n}$ & \multicolumn{1}{c}{$\%$} & Masc. & $\%$ \\
\hline$<1$ & 7 & 7,4 & 6 & 85,7 \\
$1-3$ & 33 & 35,1 & 25 & 75,8 \\
$3-6$ & 10 & 10,6 & 9 & 83,3 \\
$6-11$ & 24 & 24,5 & 17 & 68 \\
$11-15$ & 14 & 14,9 & 9 & 64,3 \\
$>15$ & 7 & 7,4 & 5 & 71,4 \\
\hline Total & 94 & 100 & 71 & 75,53 \\
\hline
\end{tabular}

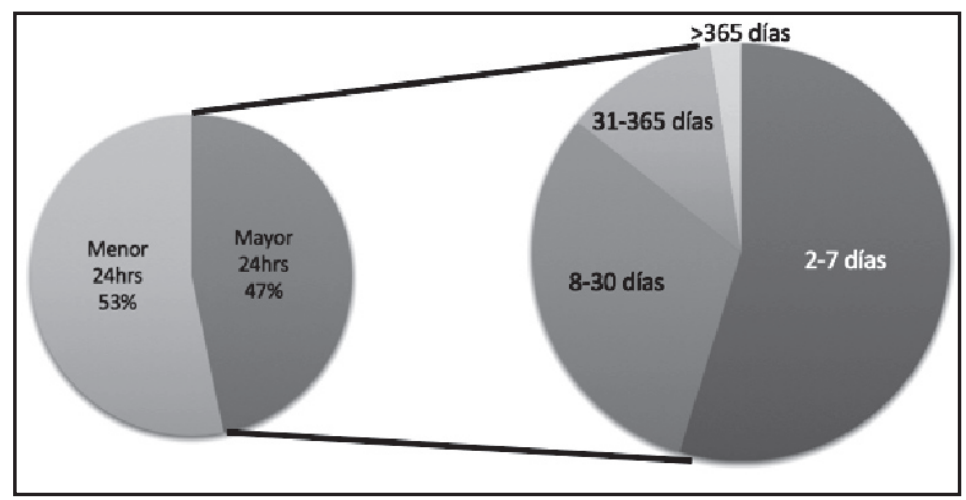

Figura 1. Tiempo tomado hasta el diagnóstico. 
Tabla 2. Detalle de los CEVA encontrados

\begin{tabular}{|c|c|c|}
\hline Cuerpo extraño & $n$ & $\%$ \\
\hline Maní & 25 & 26,5 \\
\hline Chinche & 12 & 12,7 \\
\hline Tapa de lápiz & 10 & 10,6 \\
\hline Alfiler & 7 & 7,4 \\
\hline Plástico & 4 & 4,2 \\
\hline Poroto & 4 & 4,2 \\
\hline Diente & 4 & 4,2 \\
\hline Piedra & 3 & 3,2 \\
\hline Goma & 3 & 3,2 \\
\hline Nuez & 3 & 3,2 \\
\hline Pito & 2 & 2,1 \\
\hline Hueso & 2 & 2,1 \\
\hline Manzana & 2 & 2,1 \\
\hline Piñón & 1 & 1,1 \\
\hline Resorte & 1 & 1,1 \\
\hline Clavo & 1 & 1,1 \\
\hline Concha & 1 & 1,1 \\
\hline LED & 1 & 1,1 \\
\hline Almendra & 1 & 1,1 \\
\hline Grapa & 1 & 1,1 \\
\hline Cierre & 1 & 1,1 \\
\hline Medicamento & 1 & 1,1 \\
\hline Algodón & 1 & 1,1 \\
\hline Mica de reloj & 1 & 1,1 \\
\hline Tornillo & 1 & 1,1 \\
\hline Sin datos & 1 & 1,1 \\
\hline Total & 94 & 100 \\
\hline
\end{tabular}

tomías en el $4 \%$ de los casos (dos porotos, una piedra y una tapa de lápiz).

Afortunadamente no se reportaron complicaciones durante el proceso de la BR ni muertes que lamentar.

\section{DISCUSIÓN}

La falla en el diagnóstico de CEVA se ha reportado entre 20\%-25\% según dos grandes casuísticas ${ }^{7-8}$. En nuestra revisión el $29,8 \%$ de los casos consultó más de una vez previo al diagnóstico de CEVA, y sólo en el $53 \%$ de los casos el diagnóstico se realizó dentro de las primeras 24 horas. Dentro de las posibles causas, la más importante es una historia poco clara, sobre todo en la edad más frecuente que es antes de los tres años, donde los niños no pueden/saben expresarse bien. También puede suceder que algunos médicos de urgencia subestimen lo ocurrido cometiendo errores por falta de experiencia en este accidente que no tiene una historia ni síntomas patognomónicos, ni siquiera el síndrome de sofocación, que en nuestra revisión fue poco específico. Muchas veces estos pacientes son tratados como cuadros de laringitis aguda, asma, neumonías o infecciones respiratorias altas, retrasando el diagnóstico y aumentando las complicaciones ${ }^{9}$, que explica en nuestra revisión un grupo no despreciable de casos donde el diagnóstico se realizó después del mes de ocurrida la aspiración. Lo anterior es una realidad a nivel mundial según un metaanálisis que reunió 1.063 artículos en relación a CEVA ${ }^{10}$.

Como técnica diagnóstico-terapéutica, la BR es el gold standard ya que permite un manejo seguro de la vía aérea, sin embargo, sólo como herramienta diagnóstica involucra los riesgos asociados al procedimiento en sí y de la anestesia. La radiografía de tórax (RxTx), ampliamente disponible y de fácil acceso, es altamente sensible en los casos que el CEVA es radioopaco. En nuestra revisión el 74\%

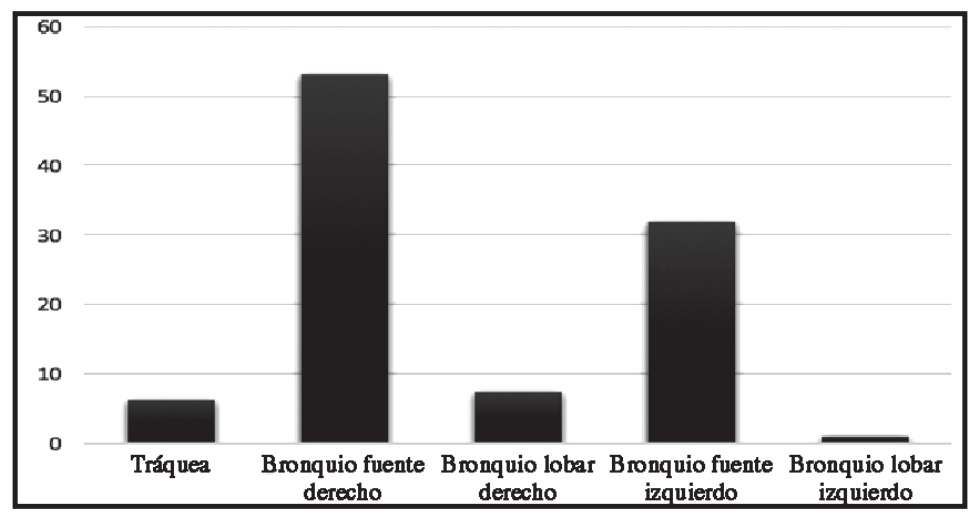

Figura 2. Distribución de los CEVA en el árbol bronquial (\%). 
de los casos fueron radiolúcidos, por tanto cobran importancia los signos indirectos como atelectasias, signos de atrapamiento aéreo, condensaciones, ensanchamiento mediastínico, enfisema, etc. En la presente revisión, y de forma interesante, el 80,9\% de Ios CEVA radiolúcidos presentaron alguna alteración en la RxTx versus $39,1 \%$ de los casos en que la BR fue negativa para CEVA (hallazgos descritos en ficha clínica previo a la extracción), siendo esta diferencia estadísticamente significativa, con una sensibilidad del $83 \%$ y una especificidad del $61 \%$. Estos datos son similares a lo reportado en otras revisiones ${ }^{9}$. Por otra parte, durante los últimos años la tomografía computarizada de tórax (TCTx) se ha estado posicionando como una técnica diagnóstica no invasiva y útil. Los hallazgos que se pueden observar son el CEVA propiamente tal 0 algún bloqueo en la vía aérea, en un bronquio fuente, segmentario e incluso subsegmentario ${ }^{11,12}$. En nuestro centro, la TCTx no es un procedimiento de rutina en el proceso diagnóstico, reservándose sólo para los casos donde la sospecha diagnóstica es pobremente fundada. Para nuestro equipo, aun con un TCTx normal, si la sospecha diagnóstica es importante de todas formas debe realizarse la broncoscopía rígida.

En la Figura 3 se ofrece un algoritmo sugerido de acción frente a la sospecha de CEVA.
En relación a la edad de presentación, en nuestra revisión detectamos un predominio en menores de 3 años, pero no significativamente respecto de otros grupos etarios. Lo anterior difiere de publicaciones extranjeras ${ }^{8,13-15}$, la última revisión realizada en nuestro hospital el año 1984 y de la última serie publicada en Chile el año 1953, pero es similar al metaanálisis anteriormente mencionado ${ }^{10}$. Encontramos un segundo peak de incidencia en el grupo de seis a once años, con un fuerte predominio de CEVA inorgánicos, especialmente tapas de lápices y chinches, a diferencia del grupo de menores de tres años, donde el maní constituyó el CEVA por excelencia con un $p<0,05$ respecto a los otros grupos de edad, similar a otros reportes ${ }^{10}$. Siempre predominó el sexo masculino, similar a lo reportado previamente en muchas series, posiblemente por la personalidad más intrusiva de éstos respecto de las niñas.

La ubicación de preferencia en el árbol bronquial derecho continúa repitiéndose en nuestra serie y en diferentes investigaciones internacionales. Esto no debería cambiar dada la especial anatomía del bronquio fuente derecho que lo hace más susceptible, y por tanto, no requiere de mayor análisis en esta discusión.

El éxito de la extracción mediante la BR fue de $96,8 \%$ lo cual está acorde con la literaturar-3,7,8,14-16.

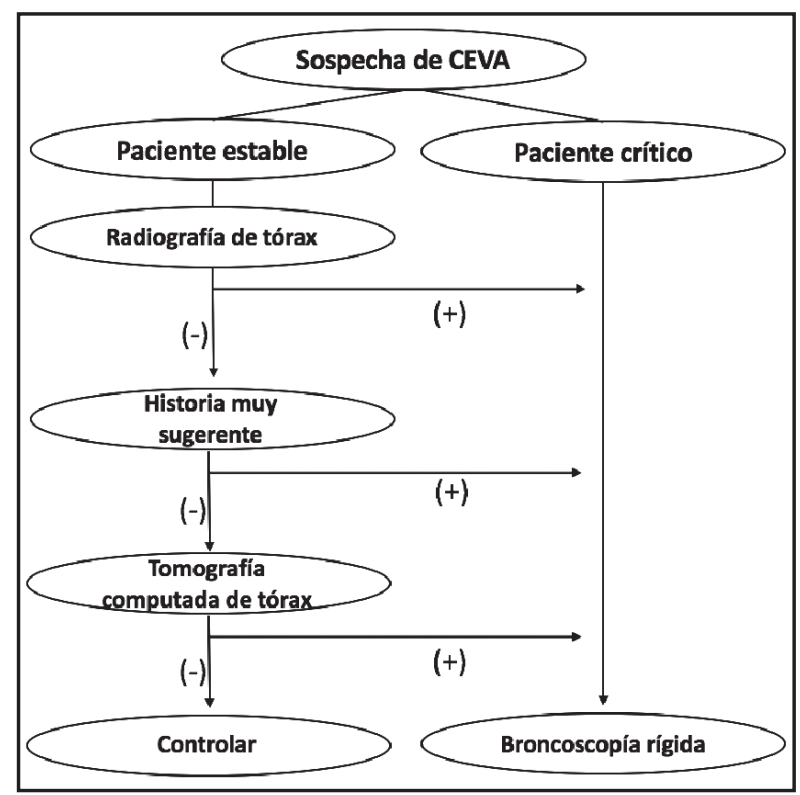

Figura 3. Algoritmo de procedimiento sugerido. 
Por fortuna no tuvimos complicaciones relevantes. En siete pacientes (7,4\%) hubo que extraer el CEVA en un segundo intento, lo cual es algo por sobre el 0,97\%-4,4\% encontrado ${ }^{14,15}$. En tres casos $(3,2 \%)$ hubo que realizar toracotomía, Io cual concuerda con la literatura $1,7,14,15$. No tuvimos muertes que lamentar.

\section{CONCLUSIONES}

El diagnóstico de CEVA es difícil dado que sus síntomas muchas veces se confunden con asma 0 infecciones respiratorias, lo que resulta en retrasos en el diagnóstico y su tratamiento. Aunque la mortalidad por CEVA es baja, el trabajo conjunto entre pediatras, radiólogos y otorrinolaringólogos es esencial para un diagnóstico y tratamiento oportuno. La RxTx continúa siendo de utilidad en presencia de una historia compatible con aspiración de cuerpo extraño, pero si esta última o el TCTx no evidencian CEVA y la historia es sugerente, la BR debería realizarse.

\section{BIBLIOGRAFÍA}

1. Cheng W, Tam P. Foreign-body ingestion in children: Experience with 1,265 cases. J Pediatr Surg 1999; 34: 1472-6.

2. Otte J, Girardi G, Contador A. Errores en el diagnóstico y manejo del cuerpo extraño en la vía aérea. Rev Otorrinolaringología Cir Cabeza Cuello 1995; 55: 13-24.

3. BordagaRAY P. Cuerpos extraños de la vía inferior. Rev Otorrinolaringología Cir Cabeza y Cuello 1988; 61-4.

4. Andreoli et al. Is inpatient admission necessary following removal of airway foreign bodies? International Journal of Pediatric Otorhinolaryngology 2015; 79: 1436-8.

5. Bejar P, Marco et al. Mortalidad nacional en otorrinolaringología. Rev Otorrinolaringol Cir Cabeza Cuello 2007; 67: 31-7.
6. Sersar S, Rizk W, Bilal M, ElDiasty M, Eltantawy $T$, AB- DelhaKam B. Inhaled foreign bodies: presentation, management and value of history and plain chest radiography in delayed presentation. Otolaryngol Head Neck Surg 2006; 134: 92-9.

7. Cataneo a, Cataneo D, Ruiz Jr R. Management of tracheobronchial foreign body in children. Pediatr Surg Int 2008; 24: 151-6.

8. Gang W, Zhengxia P, Hongbo L, Yonggang L, Jiangtao D, Shengde W, Chun W. Diagnosis and treatment of tracheobronchial foreign bodies in 1024 children. Journal of Pediatric Surgery 2012; 47: 2004-10.

9. Kiyan G, Gocmen B, Tugtepe H, Karakoc F, Dagli E, DAGLI TE. Foreign body aspiration in children: the value of diag- nostic criteria. Int J Pediatr Otorhinolaryngol 2009; 73: 963-7.

10. Foltran F et al. Foreign bodies in the airways: A meta-analysis of published papers. International Journal of Pediatric Otorhinolaryngology 2012; 76S: 512-9.

11. Torres de Amorim e Silva C, Fink A. Case 137: pneumonia and bronchiectasis secondary to unrecognized peanut impaction. Radiology 2008; 248: 1080-2.

12. ShILPA ET AL. Tracheobronchial Foreign Bodies in Children: Imaging Assessment. Semin Ultrasound CT MRI 2014; 36: 8-20.

13. Adaletli I, Kurugoglu S, Ulus S, et al. Utilization of low-dose multidetector CT and virtual bronchoscopy in children with suspected foreign body aspiration. Pediatr Radiol 2007; 37: 33-40.

14. Tang F, Chen M, Du Z, et al. Fibrobronchoscopic treatment of foreign body aspiration in children: an experience of 5 years in Hangzhou City, China. J Pediatr Surg 2006; 41: e1-5.

15. Saki N, Nikakhlagh S, Mohammad S. 25-Year Review of the Abundance and Diversity of Radiopaque Airway Foreign Bodies in Children. Indian J Otolaryngol Head Neck Surg 2015; 67(3): 261-6.

16. PANDA ET AL. Foreign body in the bronchus in children: 22 years experience in a tertiary care paediatric centre. Afr J Paediatr Surg 2014; 11(3): 252-5.

Dirección: Ignacio Mendoza A.

Servicio de Otorrinolaringología y Cirugía de Cabeza y Cuello

Hospital Guillermo Grant Benavente

San Martín 1436

E mail: drignaciomendoza@gmail.com 